\title{
Relação entre Velocidade de Onda de Pulso e Biomarcadores Cardiovasculares em Pacientes com Fatores de Risco
}

\author{
Relationship between Pulse Wave Velocity and Cardiovascular Biomarkers in Patients with Risk Factors \\ Rayne Ramos Fagundes, ${ }^{1 \oplus}$ Priscila Valverde Oliveira Vitorino, ${ }^{2}$ Ellen de Souza Lelis, ${ }^{2}$ Paulo Cesar B. Veiga Jardim, ${ }^{3 \oplus}$ \\ Ana Luiza Lima Souza, ${ }^{1,3}$ Thiago de Souza Veiga Jardim, ${ }^{1,3}{ }^{10}$ Pedro Miguel Guimarães Marques Cunha, ${ }^{4}$ Weimar Kunz \\ Sebba Barroso ${ }^{1,3}$ (D) \\ Universidade Federal de Goiás, ${ }^{1}$ Goiânia, GO - Brasil \\ Pontifícia Universidade Católica de Goiás - Escola de Ciências Sociais e da Saúde, ${ }^{2}$ Goiânia, GO - Brasil \\ Universidade Federal de Goiás - Liga de Hipertensão, ${ }^{3}$ Goiânia, GO - Brasil \\ Universidade do Minho Escola de Medicina, ${ }^{4}$ Braga - Portugal
}

\section{Resumo}

Fundamento: A relação entre velocidade de onda de pulso (VOP) e biomarcadores de mudanças estruturais do ventrículo esquerdo e artérias carótidas ainda é pouco elucidada.

Objetivo: Investigar a relação entre VOP e esses biomarcadores.

Métodos: Estudo transversal, retrospectivo e analítico. Revisamos prontuários médicos de pacientes com diabetes mellitus, dislipidemia, e pré-hipertensão ou hipertensão, que realizaram medida de pressão arterial central (PAC) utilizando o Mobil-O-Graph ${ }^{\circledR}$, e doppler de carótida ou ecocardiografia três meses antes ou após a medida da PAC. Análise estatística realizada por correlação de Pearson ou de Spearman, análise de regressão múltipla e de regressão bivariada, e teste $t$ (independente) ou de Mann-Whitney. Um p <0,05 indicou significância estatística.

Resultados: Prontuários de 355 pacientes foram avaliados, 56,1 $\pm 14,8$ anos, 51\% homens. A VOP correlacionou-se com espessuras da íntima média (EIM) das carótidas $(r=0,310)$ do septo do ventrículo esquerdo $(r=0,191)$ e da parede posterior do ventrículo esquerdo $(r=0.215)$, e com diâmetro do átrio esquerdo $(r=0,181)$. A EIM associou-se com VOP ajustada por idade e pressão sistólica periférica $(p=0,0004)$; uma EIM maior que $1 \mathrm{~mm}$ aumentou em 3,94 vezes a chance de se apresentar VOP acima de $10 \mathrm{~m} / \mathrm{s}$. A VOP foi significativamente maior em indivíduos com hipertrofia do ventrículo esquerdo $(p=0,0001)$, EIM $>1 \mathrm{~mm}(p=0,006)$, placa de carótida $(p=0,0001)$, estenose $\geq 50 \%(p=0,003)$, e lesões de órgãos-alvo $(p=0,0001)$.

Conclusões: A VOP correlacionou-se com a EIM e com parâmetros ecocardiográficos, e se associou independentemente com EIM. Essa associação foi mais forte em pacientes com hipertrofia do ventrículo esquerdo, EIM aumentada, placa de carótida, estenose $\geq 50 \%$, e lesões de órgãos-alvo. (Arq Bras Cardiol. 2020; 115(6):1125-1132)

Palavras-chave: Doenças Cardiovasculares/mortalidade; Pressão Arterial; Fatores de Risco; Hipertensão; Disfunção Ventricular Esquerda; Diabetes Mellitus.

\footnotetext{
Abstract

Background: The relationship between pulse wave velocity (PWV) and biomarkers of structural changes of the left ventricle and carotid arteries remains poorly understood.

Objective: To investigate the relationship between PWV and these biomarkers.

Methods: This was an analytical, retrospective, cross-sectional study. Medical records of patients with diabetes mellitus, dyslipidemia, and pre-hypertension or hypertension, who underwent central blood pressure (CBP) measurement using Mobil-O-Graph $\AA_{\text {, and carotid doppler }}$ or echocardiography three months before and after the CBPM were analyzed. Statistical analysis was performed using Pearson or Spearman correlation, linear bivariate and multiple regression analysis, and the $t$ test (independent) or Mann-Whitney test. A $p<0.05$ indicated statistical significance.
}

Correspondência: Rayne Ramos Fagundes

Universidade Federal de Goiás - R. 235, s/n. CEP 74605-220 - Setor Leste Universitário, Goiânia, GO - Brasil

E-mail: rayneramos.f@gmail.com, rayne_rf@yahoo.com.br

Artigo recebido em 08/01/2019; revisado recebido em 30/08/2019; aceito em 08/10/2019

DOI: https://doi.org/10.36660/abc.20190348 
Results: Medical records of 355 patients were analyzed, mean age 56.1 ( \pm 14.8 ) years, 51\% male. PWV was correlated with intima-media thickness (IMT) of carotids ( $r=0.310)$ and left ventricular septal thickness $(r=0.191)$, left ventricular posterior wall thickness $(r=0.215)$, and left atrial diameter $(r=0.181)$. IMT was associated with PWV adjusted by age and peripheral systolic pressure ( $p=0.0004) ; \mathrm{IMT}$ greater than $1 \mathrm{~mm}$ increased the chance of having PWV above $10 \mathrm{~m} / \mathrm{s}$ by 3.94 times. PWV was significantly higher in individuals with left ventricular hypertrophy $(p=0.0001), I M T>1 \mathrm{~mm}(p=0.006)$, carotid plaque $(p=0.0001)$, stenosis $\geq 50 \%(p=0.003)$, and target-organ damage $(p=0.0001)$.

Conclusion: PWV was correlated with IMT and echocardiographic parameters, and independently associated with IMT. This association was stronger in individuals with left ventricular hypertrophy, increased IMT, carotid plaque, stenosis $\geq 50 \%$, and target organ damage. (Arq Bras Cardiol. 2020; 115(6):1125-1132)

Keywords: Cardiovasclar Diseases/mortality; Blood Pressure; Risk Factors; Hypertension; Left Ventricle Dysfunction;Diabetes Mellitus.

Full texts in English - http://www.arquivosonline.com.br

\section{Introdução}

A alta prevalência e a elevada mortalidade das doenças cardiovasculares (DCVs) destacam a urgente necessidade de se implementar ferramentas para melhor estratificação de risco cardiovascular, identificar os pacientes em alto risco, e diagnosticar e tratar precocemente as doenças. Uma dessas ferramentas são os biomarcadores cardiovasculares, os quais conseguem detectar as DCVs em sua fase subclínica, com boa acurácia, melhorando, assim, a prevenção de eventos e o cenário epidemiológico. ${ }^{1,2}$

Alguns dos principais biomarcadores relacionados à estrutura e à função vascular são a espessura da íntima-média (EIM), presença de placas na artéria carótida, velocidade de onda de pulso (VOP), e o índice tornozelo-braquial (ITB). ${ }^{2}$ Além disso, outros biomarcadores cardiovasculares são usados para identificar lesões de órgãos-alvo (LOA), tais como hipertrofia do ventrículo esquerdo, níveis elevados de creatinina sérica, excreção aumentada de albumina, e taxa de filtração glomerular reduzida.,

A VOP, um marcador de dano vascular utilizado na avaliação de rigidez arterial, é considerada um forte marcador independente de LOA e eventos adversos. ${ }^{5}$ A VOP também é um preditor de mortalidade por todas as causas, indicando o risco real do paciente. ${ }^{6} \mathrm{O}$ aumento de um metro por segundo $(1 \mathrm{~m} / \mathrm{s})$ na VOP leva a um aumento de $14 \%$ no risco de eventos adversos e de $15 \%$ no risco cardiovascular e mortalidade por todas as causas. ${ }^{6}$ Entre suas vantagens, a VOP é um método não invasivo, fácil, de custo relativamente baixo, e amplamente validado, ${ }^{2}$ com valores de referência claramente estabelecidos. ${ }^{7,8}$ Apesar dessas evidências, a VOP continua subutilizada na prática clínica, e poucos estudos analisaram sua relação com outros biomarcadores, especialmente utilizando o método oscilométrico. Assim, o objetivo deste estudo foi investigar a relação entre a VOP e outros biomarcadores das alterações estruturais cardiovasculares em pacientes com fatores de risco cardiovascular.

\section{Métodos}

\section{Participantes}

De setembro de 2012 a março de 2017, foram realizadas 660 medidas da pressão arterial central (PAC). Entre essas avaliações, 131 pacientes realizaram o exame duas vezes ou mais, totalizando 169 avaliações repetidas. Assim, a população do estudo foi composta por 491 pacientes que realizaram avaliações da PAC.
O cálculo da amostra baseou-se em um erro de $5 \%$ e nível de confiança de 95\%, que indicou um tamanho amostral mínimo de 216 pacientes. A amostra do estudo consistiu em 355 pacientes brasileiros encaminhados a uma clínica de cardiologia para avaliação da PAC (Figura 1).

\section{Delineamento do Estudo e Procedimentos}

Este estudo analítico, retrospectivo, transversal foi realizado a partir da análise de prontuários médicos e laudos de exames. Dados foram primeiramente coletados dos prontuários médicos do serviço de arquivo médico da instituição. Foram aplicados os seguintes critérios de exclusão: idade inferior a 18 anos, ausência dos seguintes diagnósticos: diabetes mellitus (DM), dislipidemia (DLP), pré-hipertensão $(\mathrm{PH})$ ou hipertensão (HT); ausência de Doppler de carótida ou de ecocardiografia nos três meses antecedentes ou posteriores à medida de PAC (Figura 1).

Os diagnósticos de todos os pacientes foram obtidos dos prontuários médicos e, quando esses não estavam disponíveis, os seguintes critérios diagnósticos foram usados - glicemia de jejum > $125 \mathrm{mg} / \mathrm{dL}$ ou uso de hipoglicemiantes para DM, níveis de triglicerídeos $>150 \mathrm{mg} / \mathrm{dL}$ e de lipoproteína de baixa densidade $(\mathrm{LDL})>100 \mathrm{mg} / \mathrm{dL}$ e/ou de lipoproteína de alta densidade (HDL) $<40 \mathrm{mg} / \mathrm{dL}$ e/ou uso atual de estatinas para dislipidemia. Indivíduos com pressão arterial sistólica (PAS) periférica entre 121 e 139 mmHg e pressão arterial diastólica (PAD) entre 81 e $89 \mathrm{mmHg}$, medidas durante a avaliação da PAC, foram classificados como pré-hipertensos, e aqueles com pressão arterial igual ou superior a 140/90 $\mathrm{mmHg}$ foram classificados como hipertensos. ${ }^{4}$

Dados sobre as seguintes variáveis foram coletadas dos prontuários médicos: sexo (feminino ou masculino), tabagismo (sim ou não), e estado civil (com ou sem parceiro/a). Além dos resultados dos exames de imagem, resultados do Doppler de carótida e/ou ecocardiografia conduzidos nos três meses antes e após o exame de PAC foram analisados. Quando esses exames eram realizados mais de uma vez nesse período, os resultados do último teste antes da medida da PAC foram considerados para análise.

\section{Medida da PAC}

A PAC foi determinada pelo método validado, nãoinvasivo, oscilométrico, pelo equipamento Mobil-O-Graph ${ }^{\circledR}$ (IEM, Stolberg, Alemanha), com algoritmo ARCSolver. ${ }^{10}$ Todas as medidas foram realizadas pelo mesmo indivíduo, 


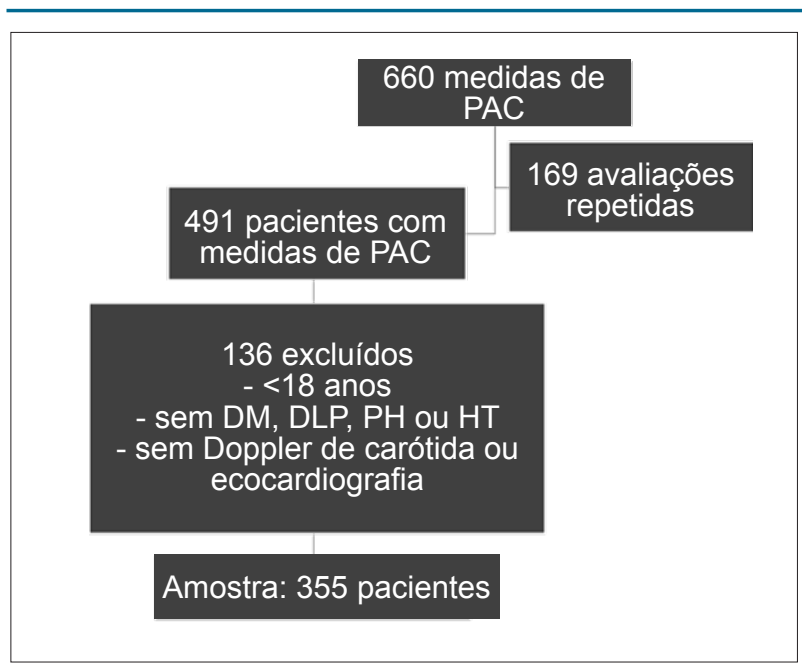

Figura 1 - Fluxograma da seleção da amostra do estudo; PAC: pressão arterial central; DM: diabetes mellitus; $P H$ : pré-hipertensão; HT: hipertensão.

sempre das 13 horas às 14 horas, utilizando a análise tripla de onda de pulso e calibração MAD-c2 (pressão diastólica média). ${ }^{9,10}$ Idade foi calculada como a diferença entre a data de nascimento e a data da medida da PAC. Peso $(\mathrm{Kg})$ e altura (m) foram usados para o cálculo do índice de massa corporal (IMC, $\mathrm{Kg} / \mathrm{m}^{2}$ ) pela fórmula de Quetelet ${ }^{11}$ e sua classificação. ${ }^{12}$ PAS periférica (PASp), PAD periférica (PADp), PAS central (PASc), índice de aumento (IA), e VOP foram analisados. ${ }^{13}$ Todos os pacientes foram orientados a não fumar ou beber café antes do teste.

\section{Doppler de Carótida e Ecocardiograma}

Os exames de imagem foram realizados em diferentes centros de imagem, segundo escolha do paciente. Exames realizados na clínica de cardiologia em que ocorreu a coleta de dados foram conduzidos com equipamento de ultrassom Philips HD 11.

O Doppler de carótida foi realizado seguindo-se as diretrizes norte-americanas ${ }^{14}$ e europeia. ${ }^{15}$ Os valores mais altos obtidos das artérias carótidas comuns direita e esquerda foram considerados para a análise estatística.

Os parâmetros ecocardiográficos foram avaliados por ecocardiografia transtorácica bidimensional, ${ }^{16}$ com medidas da espessura do septo do ventrículo esquerdo (ESVE), da espessura da parede posterior do ventrículo esquerdo (EPPVE), e do diâmetro do átrio esquerdo (DAE).

\section{Lesões de Órgãos-alvo}

A identificação de LOA baseou-se na presença de EIM aumentada, ${ }^{17}$ placas de ateroma no Doppler de carótida, ${ }^{3,4}$ hipertrofia do ventrículo esquerdo (HVE) no ecocardiograma, ${ }^{18}$ e aumento da rigidez arterial identificado por uma VOP maior que $10 \mathrm{~m} / \mathrm{s}^{3,4}$ (Figura 2).

\section{Análise Estatística}

Os dados foram coletados e escaneados em duplicata por dois pesquisadores, utilizando o programa Epidata, versão

\begin{tabular}{|c|c|c|}
\hline Exame de imagem & $\begin{array}{l}\text { Sexo / } \\
\text { Grupo }\end{array}$ & Valores de referência \\
\hline \multirow{2}{*}{ Ecocardiografia ${ }^{17}$} & Feminino & $\begin{array}{c}\text { ESVE }>0,9 \mathrm{~mm} \\
\text { EPPVE }>0,9 \mathrm{~mm} \\
\text { DAE }>38 \mathrm{~mm}\end{array}$ \\
\hline & Masculino & $\begin{array}{l}\text { ESVE }>1,0 \mathrm{~mm} \\
\text { EPPVE }>1,0 \mathrm{~mm} \\
\text { DAE }>40 \mathrm{~mm}\end{array}$ \\
\hline \multirow{2}{*}{ Doppler de carótida } & \multirow{2}{*}{$\begin{array}{l}\text { Masculino ou } \\
\text { feminino }\end{array}$} & $\mathrm{EIM}>1 \mathrm{~mm}^{16}$ \\
\hline & & Placas de ateroma ${ }^{3,4}$ \\
\hline $\begin{array}{l}\text { Medida de pressão } \\
\text { arterial central }\end{array}$ & $\begin{array}{l}\text { Masculino ou } \\
\text { feminino }\end{array}$ & $\mathrm{VOP}>10 \mathrm{~m} / \mathrm{s}^{3,4}$ \\
\hline
\end{tabular}

Figura 2 - Exames e valores de referência considerados indicativos de lesões de órgãos-alvo. DAE: diâmetro do átrio esquerdo; EIM: espessura da intima média; EPPVE: espessura da parede posterior do ventrículo esquerdo; ESVE: espessura do septo do ventrículo esquerdo; VOP: velocidade de onda de pulso.

3.1. Após analisar e corrigir as inconsistências, os dados foram exportados para o programa Statistical Package for Social Science (SPSS), versão 18.0. O teste de Kolmogorov-Smirnov foi aplicado, e se realizou uma análise descritiva dos dados. A análise estatística foi realizada com base na distribuição dos dados, utilizando testes paramétricos e não paramétricos. Os dados numéricos foram descritos como média e desvio padrão ou média e intervalo interquartil, dependendo da distribuição dos dados. As variáveis categóricas foram apresentadas em frequência absoluta e relativa. O coeficiente de correlação de Pearson ou a correlação de Spearman foram usados para avaliar a correlação da VOP com os resultados obtidos no Doppler de carótida e ecocardiograma. As correlações foram classificadas como fraca $(0<r<0,30)$, moderada $(0,30 \leq r<$ $0,60)$, forte $(0,60 \leq r<0,90)$ e muito forte $(0,90 \leq r<1) .{ }^{19}$

A associação entre a VOP e outros biomarcadores (EIM, ESVE, EPPVE e DAE) foi avaliada por análise de regressão linear bivariada. As variáveis com $p<0.020$ foram usadas na análise de regressão múltipla. Todas as premissas para a aplicação da análise de regressão linear foram atendidas. A VOP foi comparada segundo magnitude da EIM, presença ou não de HVE, presença ou não de placa, dimensão da placa, e presença ou não de LOA, utilizando-se o teste para amostras independentes ou o teste de Mann-Whitney. Valores de $p<0,05$ foram considerados estatisticamente significativos.

\section{Aspectos Éticos}

O estudo foi conduzido de acordo com a resolução 466/12 do Conselho Nacional de Saúde, e foi aprovado pelo comitê de ética do Hospital das Clínicas da Universidade Federal de Goiás (UFG) (protocolo de aprovação número 1.500.463).

\section{Resultados}

Um total de 355 indivíduos, com idade média de $56,1( \pm 14,8)$ anos participaram no estudo. A maioria apresentou dislipidemia e/ou hipertensão arterial, 148 (41;7\%) apresentavam sobrepeso e 130 (36.6\%) eram obesos (Tabela 1). 


\section{Artigo Original}

Uma correlação moderada e positiva foi encontrada entre a VOP e a EIM; e correlações positivas fracas foram identificadas entre a VOP e a ESVE, e entre a EPPVE e DAE) (Tabela 2).

A EIM foi associada com a VOP ajustada para idade e pressão sistólica periférica $(p=0.0004)$. Um aumento de $1 \mathrm{~mm}$ ou mais na EIM aumentou em 3.94 vezes a chance de se apresentar VOP acima de 10m/s (Tabelas 3 e 4).

A VOP foi significativamente maior em indivíduos com HVE, com EIM mais elevada, e indivíduos com placa de carótida, estenose igual ou maior que 50\%, e com LOA (Tabela 5).

\section{Discussão}

No presente estudo, a VOP correlacionou-se com todos os biomarcadores avaliados, e se associou com EIM, mesmo após ajuste para idade e pressão sistólica periférica. A chance de apresentar VOP acima de $10 \mathrm{~m} / \mathrm{s}$ foi 3,94 vezes maior na presença de EIM maior que $1 \mathrm{~mm}$. A VOP apresentou um aumento linear com a presença e tamanho da placa de ateroma, e com a presença de LOA. Esses resultados estão de acordo com os de estudos publicados anteriormente, ${ }^{2,20,21}$

Tabela 1 - Características da amostra

\begin{tabular}{|c|c|}
\hline Variáveis & $\begin{array}{c}\text { Média (DP) / Mediana } \\
(25 \%-75 \%) / \mathrm{n}(\%)\end{array}$ \\
\hline Idade & $56,1( \pm 14,8)$ \\
\hline IMC & $28,7( \pm 4,9)$ \\
\hline PASc & $113(107-123)$ \\
\hline IA & $21,5( \pm 13,4)$ \\
\hline VOP & $8,2( \pm 2)$ \\
\hline \multicolumn{2}{|l|}{ Sexo } \\
\hline Masculino & $181(51 \%)$ \\
\hline Feminino & $174(49 \%)$ \\
\hline \multicolumn{2}{|l|}{ Estado civil } \\
\hline Com parceiro & $251(70,7 \%)$ \\
\hline Sem parceiro & $102(28,7 \%)$ \\
\hline \multicolumn{2}{|c|}{ Fatores de risco cardiovasculares } \\
\hline Sobrepeso & $148(41.7 \%)$ \\
\hline Obesidade & $130(36,6 \%)$ \\
\hline Tabagismo & $12(3,4 \%)$ \\
\hline \multicolumn{2}{|l|}{ Diagnóstico } \\
\hline Dislipidemia & $306(86,2 \%)$ \\
\hline Hipertensão arterial & $283(79,7 \%)$ \\
\hline Diabetes mellitus & $65(18.3 \%)$ \\
\hline Pré-hipertensão & $47(13,2 \%)$ \\
\hline
\end{tabular}

IA: índice de aumento; IMC: índice de massa corporal; VOP: velocidade de onda de pulso; PASc: pressão arterial sistólica central.
Tabela 2 - Correlação da velocidade de onda de pulso e biomarcadores cardiovasculares

\begin{tabular}{cccccc}
\hline & & $\begin{array}{c}\text { EIM } \\
(n=178)\end{array}$ & $\begin{array}{c}\text { ESVE } \\
(n=313)\end{array}$ & $\begin{array}{c}\text { EPPVE } \\
(n=312)\end{array}$ & $\begin{array}{c}\text { DAE } \\
(n=312)\end{array}$ \\
\hline \multirow{3}{*}{ VOP } & $r$ & $0,310^{\dagger}$ & $0,191^{\dagger}$ & $0,215^{\dagger}$ & $0,181^{\ddagger}$ \\
\cline { 2 - 6 } & $p$ & $<0,001^{*}$ & $0,001^{*}$ & $<0,001^{*}$ & $0,001^{*}$ \\
\hline
\end{tabular}

${ }^{*} p$ < 0.05. †Correlação de Spearman; łCorrelação de Pearson; EIM: espessura da intima média; DAE: diâmetro do átrio esquerdo; EPPVE: espessura da parede posterior do ventrículo esquerdo; ESVE: espessura do septo ventricular esquerdo; VOP: velocidade de onda de pulso.

Tabela 3 - Análise de regressão linear bivariada da velocidade de onda de pulso com biomarcadores cardiovasculares

\begin{tabular}{llcc}
\hline Variáveis & OR & IC95\% (OR) & p \\
\hline ESVE & 2,49 & $1,38-4,49$ & $0,003^{*}$ \\
\hline EIM & 3,94 & $1,53-10,15$ & $0,004^{*}$ \\
\hline EPPVE & 2,34 & $1,29-4,22$ & $0,005^{*}$ \\
\hline DAE & 2,55 & $1,18-5,49$ & $0,017^{*}$ \\
\hline $\begin{array}{l}\text { IC: intervalo de confiança; EIM: espessura da intima média; DAE: diâmetro } \\
\text { do átrio esquerdo; EPPVE: espessura da parede posterior do ventrículo } \\
\text { esquerdo; ESVE: } \\
\text { velocidade de onda de pulso; OR: odds ratio; * } p<0,05 .\end{array}$
\end{tabular}

e reforçam o valor desse biomarcador e sua capacidade de identificar precocemente lesões cardiovasculares, além de seu excelente custo-benefício.

A correlação da VOP com parâmetros ecocardiográficos encontrada no presente estudo pode ser explicada pelo fato de que a rigidez arterial aumenta a PAS, o que causa um retorno precoce das ondas de pulso na sístole em vez de na diástole, e aumento da pós-carga do ventrículo esquerdo. Esse aumento de carga imposto sobre o miocárdio promove hipertrofia cardíaca, e consequente hipertrofia ventricular. ${ }^{22-24}$

A HVE, a qual pode ser identificada pelo aumento na espessura da parede do ventrículo esquerdo no ecocardiograma, correlaciona-se com a VOP, e valores de VOP são significativamente maiores em indivíduos com HVE. ${ }^{22,23}$ A sobrecarga sobre o ventrículo esquerdo é uma das principais causas de eventos cardiovasculares relacionados com a PAC. ${ }^{25}$

Muitos estudos apresentam não só uma correlação ${ }^{26-28}$ como também uma associação entre rigidez arterial e HVE. ${ }^{22,23,29-32}$ Portanto, a rigidez arterial aumentada pode ser usada como preditor de HVE, contribuindo para a prevenção e diagnóstico dessa condição. ${ }^{23}$

Em nosso estudo, não observamos uma associação independente da VOP com ESVE, EPPVE ou DAE, possivelmente por não termos realizado uma análise de associação entre hipertrofia e VOP como nos estudos citados, mas sim entre parâmetros ecocardiográficos e VOP. Ainda, em um dos estudos citados, ${ }^{22}$ foram utilizados achados eletrocardiográficos e não resultados ecocardiográficos, e 
Tabela 4 - Análise de regressão múltipla da velocidade de onda de pulso com biomarcadores cardiovasculares

\begin{tabular}{lcccccc}
\hline Variáveis & OR ajustado & IC $95 \%($ OR) & $\mathbf{p}$ & OR ajustado* & IC 95\% (OR) & p \\
\hline LVST & 1,64 & $0,59-4,5$ & 0,340 & - & - & - \\
\hline IMT & 3,94 & $1,53-10,15$ & 0,004 & 6,86 & $1,78-26,45$ & $<0,001$ \\
\hline LVPWT & 1,69 & $0,64-4,49$ & 0,294 & - & - & - \\
\hline LAD & 1,34 & $0,27-6,80$ & 0,705 & - & - \\
\hline
\end{tabular}

IC: intervalo de confiança; EIM: espessura da íntima média; DAE: diâmetro do átrio esquerdo; EPPVE: espessura da parede posterior do ventriculo esquerdo; ESVE: espessura do septo ventricular esquerdo; VOP: velocidade de onda de pulso; OR: odds ratio; * $p<0,05$.

Tabela 5 - Comparação da velocidade de onda de pulso de acordo com variáveis de Doppler de carótida e presença ou não de lesões de órgãos-alvo

\begin{tabular}{|c|c|c|c|c|c|}
\hline Variável & Grupo & $\mathrm{n}$ & VOP & IC & $\mathrm{p}$ \\
\hline \multirow{2}{*}{$\mathrm{HVE}^{\dagger}$} & Não & 212 & 7,6 & $7,55-8,03$ & \multirow{2}{*}{$<0,0001^{*}$} \\
\hline & Sim & 105 & 9,1 & $8,74-9,53$ & \\
\hline \multirow{2}{*}{$\mathrm{EIM}^{\ddagger}$} & $\leq 1 \mathrm{~mm}$ & 152 & 8,07 & $7,79-8,35$ & \multirow{2}{*}{0,006} \\
\hline & $>1 \mathrm{~mm}$ & 26 & 9,12 & $8,32-9,90$ & \\
\hline \multirow{2}{*}{ Presença de placa ${ }^{\ddagger}$} & Não & 82 & 7,44 & $7,14-7,75$ & \multirow{2}{*}{$<0,0001^{*}$} \\
\hline & Sim & 172 & 9,09 & $8,83-9,35$ & \\
\hline \multirow{2}{*}{ Tamanho da placa ${ }^{\ddagger}$} & $<50 \%$ & 146 & 8,92 & $8,64-9,20$ & \multirow{2}{*}{0,003} \\
\hline & $\geq 50 \%$ & 25 & 10,0 & $9,42-10,63$ & \\
\hline \multirow{2}{*}{ Lesões de órgãos-alvo*, } & Não & 118 & 6,9 & $6,62-7,12$ & \multirow{2}{*}{$<0,0001^{*}$} \\
\hline & Sim & 237 & 8,9 & $8,69-9,17$ & \\
\hline
\end{tabular}

IC: intervalo de confiança; EIM: espessura da intima média; HVE: hipertrofia do ventrículo esquerdo; VOP: velocidade de onda de pulso; ${ }^{*} p<0,05 .{ }^{\dagger}$ Teste de Mann-

Whitney. ${ }^{\ddagger}$ Teste t para amostras independentes. ${ }^{* \star}$ EIM>1mm, presença de placa, HVE ou VOP $>10 \mathrm{~m} / \mathrm{s}$.

a maioria dos estudos realizou essa análise de associação com base no índice da massa ventricular esquerda. ${ }^{23,28,30,32}$

A relação entre o aumento da rigidez arterial e o aumento da EIM pode ser explicada pela fisiopatologia da rigidez arterial, que engloba mudanças na matriz extracelular da camada média (túnica média), incluindo quebra de elastina, depósito de colágeno, e reticulação. ${ }^{24,33}$ Tais alterações morfológicas também estão relacionadas com envelhecimento vascular. ${ }^{34}$

Um aumento da EIM também está associado com a presença de fatores de risco para arteriosclerose; e idade, pressão arterial, lipídios séricos, e níveis de glicemia de jejum são todos preditores independentes de aterosclerose de carótida. ${ }^{35}$ EIM aumentada é uma das manifestações subclínicas da arteriosclerose. ${ }^{36}$ Existe uma associação independente entre a presença de múltiplos fatores de risco cardiovasculares com aumento na EIM e redução da complacência arterial. ${ }^{37}$

A correlação ${ }^{38}$ e a associação da EIM ${ }^{38,39}$ com a VOP também foram previamente relatadas na população idosa.

A avaliação da EIM e da VOP pode aumentar a reclassificação de risco, e esses biomarcadores podem ser utilizados na identificação de LOA. ${ }^{40}$ A combinação desses biomarcadores aumenta o poder preditivo de eventos cardiovasculares em idosos, fornecendo novas informações clínicas importantes. ${ }^{41}$
Em nosso estudo, valores significativamente maiores de VOP foram identificados em indivíduos com estenose igual ou maior que $50 \%$. Valores mais elevados da VOP também se associaram significativamente com presença de placas de carótida. ${ }^{36}$ Além disso, uma redução na elasticidade da carótida está associada com presença de placas e risco de acidente vascular cerebral. ${ }^{42}$

A avaliação combinada de EIM e presença de placas melhora a predição de risco cardiovascular, e a avaliação quantitativa de placas aumenta ainda mais a sensibilidade preditiva. ${ }^{43}$ Ainda, a VOP na carótida femoral e o número de placas de ateroma estão associados de maneira significativa e independente com morte cardiovascular, e pode melhorar a identificação de indivíduos em alto risco cardiovascular. ${ }^{44}$

Além das associações entre VOP e biomarcadores, a diferença significativa na VOP encontrada entre indivíduos com e sem LOA destaca a capacidade da VOP em detectar a lesão precocemente. A rigidez arterial é um preditor independente de mortalidade tanto para diabéticos como para a população em geral, e está relacionada com desenvolvimento e progressão de LOA. ${ }^{45}$

A rigidez arterial, avaliada pela $V O P$, associa-se independentemente com a presença de LOA subclínica, 
incluindo calcificação da artéria coronária, índice tornozelo-braquial reduzido (doença arterial periférica), e hiperintensidade da substância branca (doença arterial cerebral). ${ }^{46}$

Quando a LOA está presente, mas não é identificada, muitos pacientes são erroneamente classificados como em risco baixo a médio, quando na verdade estão em um risco cardiovascular alto. ${ }^{47}$

As ferramentas diagnósticas devem ser aprimoradas e estabelecidas para a identificação precoce de um risco aumentado, para prevenir o início de LOA e suas complicações. A identificação apropriada de indivíduos com baixo risco é igualmente importante para evitar tratamentos desnecessários e seus efeitos colaterais. ${ }^{48} \mathrm{O}$ uso de biomarcadores vasculares é um método custoefetivo, com valor agregado, na melhoria da identificação de indivíduos em alto risco, facilitando, assim, a prevenção de DCV. ${ }^{44}$

As limitações deste estudo incluem: (1) quando o diagnóstico de diabetes mellitus, dislipidemia e hipertensão arterial não estava disponível nos prontuários médicos, o diagnóstico foi feito durante o estudo, de maneira ad hoc, o que pode ter subestimado ou superestimado as frequências dessas doenças. (2) Algumas variáveis de exposição também estavam ausentes nos prontuários médicos. (3) Ainda, não podemos assegurar que todos os pacientes foram submetidos ao Doppler e à ecocardiografia no mesmo local e com o mesmo avaliador. Hipertrofia não pode ser detectada pelo índice da massa ventricular, uma vez que essa informação também não estava disponível nos prontuários.

Os prontuários médicos e os critérios diagnósticos foram avaliados com rigor científico, e os dados foram revisados por dois pesquisadores, com verificação cruzada. Todos esses procedimentos devem validar nossos achados.

O presente estudo destaca a importância do uso da VOP para a detecção precoce de rigidez arterial e LOA, com foco no aumento da EIM, presença de placas de carótida, e HVE. Em geral, a VOP pode otimizar a estratificação do risco cardiovascular para facilitar a intervenção precoce e prevenir DCV e suas complicações.

\section{Conclusões}

A VOP correlacionou-se significativamente com a EIM e com parâmetros ecocardiográficos, e se associou com a EIM. Uma EIM maior que $1 \mathrm{~mm}$ aumentou a chance de se apresentar uma VOP maior que $10 \mathrm{~m} / \mathrm{s}$ em 3,94 vezes. A VOP foi maior nos indivíduos com HVE, EIM maior que $1 \mathrm{~mm}$, com estenose igual ou maior que $50 \%$, e pacientes com LOA.

\section{Contribuição dos Autores}

Concepção e desenho da pesquisa: Fagundes RR, Vitorino PVO, Barroso WKS; Obtenção de dados: Fagundes RR, Lelis ES; Análise e interpretação dos dados: Fagundes RR, Vitorino PVO, Lelis ES, Cunha PMGM, Barroso WKS; Análise estatística e Redação do manuscrito: Fagundes RR, Vitorino PVO; Revisão crítica do manuscrito quanto ao conteúdo intelectual importante: Fagundes RR, Vitorino PVO, Lelis ES, Jardim PCBV, Souza ALL, Jardim TSV, Cunha PMGM, Barroso WKS.

\section{Potencial Conflito de Interesses}

Declaro não haver conflito de interesses pertinentes.

\section{Fontes de Financiamento}

O presente estudo não teve fontes de financiamento externas.

\section{Vinculação Acadêmica}

Este artigo é parte de dissertação de Mestrado de Rayne Ramos Fagundes pela Universidade Federal de Goiás.

\section{Referências}

1. Roth GA, Johnson C, Abajobir A, Abd-Allah F, Abera SF, Abyu G, et al. Global, Regional, and National Burden of Cardiovascular Diseases for 10 Causes, 1990 to 2015. J Am Coll Cardiol.2017;70(1):1-25.

2. Vlachopoulos C, Xaplanteris P, Aboyans V, Brodmann M, Cífková R, Cosentino $F$, et al. The role of vascular biomarkers for primary and secondary prevention. A position paper from the European Society of Cardiology Working Group on peripheral circulation. Endorsed by the Association for Research into Arterial Structure and Physiology (ARTERY) Society. Atherosclerosis. 2015;241(2):507-32.

3. Mancia G, Fagard R, Narkiewicz K, Redon J, Zanchetti A, Böhm M, et al. 2013 ESH/ESC guidelines for the management of arterial hypertension: The Task Force for the management of arterial hypertension of the European Society of Hypertension (ESH) and of the European Society of Cardiology (ESC). Eur Heart J. 2013;34(28):2159-219.

4. Malachias MVB, Souza WKSB, Plavnik FL, Rodrigues CIS, Brandão AA, Neves MFT, et al. 7a Diretriz Brasileira de Hipertensão Arterial. Arq Bras Cardiol. 2016;107(Supl 3):1-83
5. Mitchell GF. Does Measurement of Central Blood Pressure have Treatment Consequences in the Clinical Praxis? Curr Hypertens Rep. 2015;17(8):1-8.

6. Vlachopoulos C, Aznaouridis K, Stefanadis C. Prediction of cardiovascular events and all-cause mortality with arterial stiffness: a systematic review and meta-analysis. J Am Coll Cardiol. 2010;55(13):1318-27.

7. The Reference Values for Arterial Stiffness' Collaboration. Determinants of pulse wave velocity in healthy people and in the presence of cardiovascular risk factors: 'establishing normal and reference values'. Eur Heart J. 2010;35(11):1367-72.

8. Díaz A, Galli C, Tringler M, Ramírez A, Cabrera Fischer El. Reference Values of Pulse Wave Velocity in Healthy People from an Urban and Rural Argentinean Population. Int J Hypertens. 2014;2014,653239

9. Jatoi NA, Mahmud A, Bennett K, Feely J. Assessment of arterial stiffness in hypertension: comparison of oscillometric (Arteriograph), piezoelectronic (Complior) and tonometric (SphygmoCor) techniques. J Hypertens. 2009;27(11):2186-91. 
10. Hametner B, Wassertheurer S, Kropf J, Mayer C, Eber B, Weber T. Oscillometric estimation of aortic pulse wave velocity: comparison with intra-aortic catheter measurements. Blood Press Monit. 2013;18(3):173-6.

11. Quelet A. Antropométrie ou mesure des différentes facultés de l'homme. . Bruxelles: C. Muquardt; 1870.

12. World Health Organization. (WHO). Physical status: The use and interpretation of anthropometry Geneva; 1995.

13. Wei W, Tolle M, Zidek W, van der Giet M. Validation of the mobil-OGraph: $24 \mathrm{~h}$-blood pressure measurement device. Blood Press Monit. 2010;15(4):225-8.

14. Stein JH, Korcarz CE, Hurst RT, Lonn E, Kendall CB, Mohler ER, et al. Use of Carotid Ultrasound to Identify Subclinical Vascular Disease and Evaluate Cardiovascular Disease Risk: A Consensus Statement from the American Society of Echocardiography Carotid Intima-Media Thickness Task Force, Endorsed by the Society for Vascular Medicine.J Am Soc Echocardiogr. 2008 2008;21(2):93-111.

15. Oates CP, Naylor AR, Hartshorne T, Charles SM, Fail T, Humphries K, et al. Joint Recommendations for Reporting Carotid Ultrasound Investigations in the United Kingdom. Eur J Vasc Endovasc Surg. 2009;37(3):251-61.

16. Lang RM, Badano LP, Mor-Avi V, Afilalo J, Armstrong A, Ernande L, et al Recommendations for cardiac chamber quantification by echocardiography in adults: an update from the American Society of Echocardiography and the European Association of Cardiovascular Imaging. J Am Soc Echocardiogr. 2015;28(1):1-39.e14.

17. Freire CMV, Alcantara ML, Santos SN, Amaral SI, Veloso O, Porto CLL, et al. Recomendação para a Quantificação pelo Ultrassom da Doença Aterosclerótica das Artérias Carótidas e Vertebrais: Grupo de Trabalho do Departamento de Imagem Cardiovascular da Sociedade Brasileira de Cardiologia - DIC -ABC Imag Cardiovasc, 2 015;28(especial):1-64.

18. Bonow RO, Mann DL, Zipes DP, Libby P. Braunwald's Heart Disease: A Textbook of Cardiovascular Medicine. United States: Elsevier Saunders; 2012.

19. Lira SA. Análise de correlação: Abordagem teórica de construção dos coeficientes com aplicações [tese]. Curitiba: Universidade Federal do Paraná; 2004.

20. Viola J, Soehnlein O. Atherosclerosis - A matter of unresolved inflammation. Semin Immunol. 2015;27(3):184-93.

21. Kotsis V, Stabouli S, Karafillis I, Nilsson P. Early vascular aging and the role of central blood pressure.J Hypertens. 2011;29(10):1847-53.

22. Chung CM, Lin YS, Chu CM, Chang ST, Cheng HW, Yang TY, et al. Arterial stiffness is the independent factor of left ventricular hypertrophy determined by electrocardiogram. Am J Med Sci. 2012;344(3):190-3.

23. Yucel C, Demir S, Demir M, Tufenk M, Nas K, Molnar F, et al. Left ventricular hypertrophy and arterial stiffness in essential hypertension. Bratis Lek Listy. 2015; 116(12):714-8.

24. Townsend RR, Wilkinson IB, Schiffrin EL, Avolio AP, Chirinos JA, CockcroftJR, et al. Recommendations for Improving and Standardizing Vascular Research on Arterial Stiffness. Hypertension. 2015;66(3):698-722.

25. Sabovic M, Safar ME, Blacher J. Is there any additional prognostic value of central blood pressure wave forms beyond peripheral blood pressure? Curr Pharmac Design. 2009;15(3):254-66

26. Pizzi O, Brandão AA, Magalhães MEC, Pozzan R, Brandão AP. Velocidade de onda de pulso - o método e suas implicações prognósticas na hipertensão arterial. RevBras Hipertens. 2006;13(1):59-62.

27. Su HM, Lin TH, Hsu PC, Lee CS, Lee WH, Chen SC, et al. Association of brachial-ankle pulse wave velocity, ankle-brachial index and ratio of brachial pre-ejection period to ejection time with left ventricular hypertrophy. Am J Med Sci. 2014;347(4):289-94.

28. Rabkin SW, Chan SH. Correlation of pulse wave velocity with left ventricular mass in patients with hypertension once blood pressure has been normalized. Heart Int. 2012;7(1):27-31.
29. Bello H, Norton GR, Ballim I, Libhaber CD, Sareli P, Woodiwiss AJ. Contributions of aortic pulse wave velocity and backward wave pressure to variations in left ventricular mass are independent of each other. J Am Soc Hypertens. 2017;11(5):265-74.

30. Masugata H, Senda S, Hoshikawa J, Murao K, Hosomi N, Okuyama H, et al. Elevated brachial-ankle pulse wave velocity is associated with left ventricular hypertrophy in hypertensive patients after stroke. Tohoku J Exper Med. 2010;220(3):177-82.

31. Park KH, Park WJ, Kim MK, Jung JH, Choi S, Cho JR, et al. Noninvasive brachial-ankle pulse wave velocity in hypertensive patients with left ventricular hypertrophy. Am J Hypertens. 2010;23(3):269-74.

32. Nitta K, Akiba T, Uchida K, Otsubo S, Otsubo Y, Takei T, et al. Left ventricular hypertrophy is associated with arterial stiffness and vascular calcification in hemodialysis patients. Hypertens Res. 2004;27(1):47-52.

33. Palombo C, Kozakova M. Arterial stiffness, atherosclerosis and cardiovascular risk: Pathophysiologic mechanisms and emerging clinical indications. Vasc Pharmacol. 2016;77:1-7.

34. Costantino S, Paneni F, Cosentino F. Ageing, metabolism and cardiovascular disease. J Physiol. 2016;594(8):2061-73.

35. Ren L, Cai J, Liang J, Li W, Sun Z. Impact of Cardiovascular Risk Factors on Carotid Intima-Media Thickness and Degree of Severity: A Cross-Sectional Study. PloS one. 2015;10(12):1-12

36. Selwaness M, van den Bouwhuijsen Q, Mattace-Raso FU, Verwoert GC Hofman A, Franco OH, et al. Arterial stiffness is associated with carotid intraplaque hemorrhage in the general population: the Rotterdam study. Arterioscler Thromb Vasc Biol. 2014:34(4):927-32.

37. Niu L, Zhang Y, Qian M, Meng L, Xiao Y, Wang Y, et al. Impact of multiple cardiovascular risk factors on carotid intima-media thickness and elasticity. PloS one. 2013;8(7):1-6.

38. Costa LS, Cunha JVL, Tress JC, Pozzan R, Neto CD, Brandão AP. A importância das medidas de pressão arterial e da velocidade da onda de pulso no desenvolvimento da hipertrofia ventricular esquerda e no espessamento médio-intimal de carótidas em pacientes idosos. Revista da SOCERJ. 2005;18(2):160-71

39. van Popele NM, Grobbee DE, Bots ML, Asmar R, Topouchian J, Reneman $\mathrm{RS}$, et al. Association between arterial stiffness and atherosclerosis: the Rotterdam Study. Stroke. 2001;32(2):454-60.

40. Bruno RM, Bianchini E, Faita F, Taddei S, Ghiadoni L. Intima media thickness, pulse wave velocity, and flow mediated dilation. Ultrassom Cardiovsc 2014;12:34.

41. Nagai K, Shibata S, Akishita M, Sudoh N, Obara T, Toba K, et al. Efficacy of combined use of three non-invasive atherosclerosis tests to predict vascular events in the elderly; carotid intima-media thickness, flow-mediated dilation of brachial artery and pulse wave velocity. Atherosclerosis. 2013;231(2):365-70

42. Boesen ME, Singh D, Menon BK, Frayne R. A systematic literature review of the effect of carotid atherosclerosis on local vessel stiffness and elasticity. Atherosclerosis. 2015;243(1):211-22.

43. Naqvi TZ, Lee MS. Carotid intima-media thickness and plaque in cardiovascular risk assessment. JACC Cardiovasc Imag. 2014;7(10):1025-38

44. Berard E, Bongard V, Ruidavets JB, Amar J, Ferrieres J. Pulse wave velocity, pulse pressure and number of carotid or femoral plaques improve prediction of cardiovascular death in a population at low risk. J Humman Hypertens. 2013;27(9):529-34

45. Prenner SB, Chirinos JA. Arterial stiffness in diabetes mellitus. Atherosclerosis $2015 ; 238(2): 370-9$.

46. Coutinho T, Turner ST, Kullo IJ. Aortic Pulse Wave Velocity Is Associated With Measures of Subclinical Target Organ Damage. JACC Cardiovascular imaging. 2011;4(7):754-61. 


\section{Artigo Original}

47. Piskorz D, Bongarzoni L, Citta L, Citta N, Citta P, Keller L, et al. World Health Organization cardiovascular risk stratification and target organ damage. Hipertens. riesgo vasc. 2016;33(1):14-20.
48. Øygarden H. Carotid Intima-Media Thickness and Prediction of Cardiovascular Disease. Am Heart J. 2017;6(1):1-3. 